\title{
Conjugated Linoleic Acid Negatively Regulates TR4 Activity in 3T3-L1 Adipocytes
}

\author{
Hojung Choi and Eungseok Kim* \\ Department of Biological Sciences, Chonnam National University, Gwangju 500-757, Korea
}

\begin{abstract}
Dietary conjugated linoleic acid (CLA) play key roles in lipid metabolism. Here, we investigated the effect of CLA on the transcriptional activity of TR4, an orphan nuclear receptor that plays an important role in lipid homeostasis. CLA increased TR4 gene mRNA level in 3T3-L1 adipocytes, but inhibited TR4 transcriptional activity in a dose-dependent manner. TR4 induced perilipin expression in 3T3-L1 adipocytes by activating perilipin promoter activity. In a gel shift assay, TR4 bound direct to the putative TR4 response element in the perilipin promoter. Interestingly, CLA reduced the interaction between TR4 and consensus DR1, a well-known TR4 binding site. Additionally, CLA inhibited TR4-induced perilipin promoter activity in a dose-dependent manner. Together, our results suggest that CLA may play a role in lipid homeostasis in adipocytes by functionally regulating TR4.
\end{abstract}

Key words: Conjugated linoleic acid, TR4, adipocytes, perilipin

\section{Introduction}

Adipose tissues are key reservoir for energy excess and properly control body lipid fluxes in response to nutrition status (Guilherme et al., 2008). Recently, adipose tissues are recognized not only as a fat storage site but also as an important endocrine organ secreting a variety of adipocytokines such as TNF $\alpha$, leptin, adiponectin and resistin. These adipocytokines have been known to participate in various physiological processes including insulin sensitivity, cell proliferation, immune response and food intake (Kershaw and Flier, 2004; Rosen and Spiegelman, 2006). Dysregulated lipid metabolism by altered expression of genes involved in lipogenesis and lipid storage increases lipid accumulation in adipocytes and consequently leads to obesity. Obesity causes insulin resistance and thus has been considered as a major risk factor for the development of diabetes, hypertension, and coronary artery disease (Gregor and Hotamisligil, 2007; Kershaw and Flier, 2004; Shi and Burn, 2004).

Conjugated linoleic acid (CLA) is a group of positional and geometric isomers of linoleic acid. The prominent

\footnotetext{
*Corresponding author: Eungseok Kim, Department of Biological Sciences, Chonnam National University, Gwangju 500757, Korea. Tel: 82-62-530-3402, Fax: 82-62-530-3409, Email: ekim@chonnam.ac.kr
}

isomers known for biological action of CLA are cis-9, trans-11 and trans-10, cis-12 CLA. Human acquires these primary isomers from milk products and ruminant meats. Several studies have shown that CLA has potential anticarcinogenic, antiatherogenic, and antiobesity properties (Ha et al., 1990; Larsen et al., 2003; Lee et al., 1994). In vitro studies also showed that trans-10, cis-12 CLA inhibited adipocyte differentiation with prevention of triglyceride (TG) accumulation in adipocytes (Kang et al., 2003; Sakuma et al., 2010). Although CLA effect on lipid accumulation in adipocytes has been reported, molecular mechanism on CLA action in adiposity remains unclear. Several reports have shown that trans-10, cis-12 CLA competitively inhibits effect of PPAR $\gamma$ agonist on PPAR $\gamma$ activity while it rather plays a role as a partial agonist of PPAR $\gamma$ in the absence of PPAR $\gamma$ agonist (Alibin et al., 2008; Granlund et al., 2003; Kennedy et al., 2009; Miller et al., 2008). However, trans-10, cis-12 CLA in the absence of PPAR $\gamma$ agonist was still able to inhibit adipocyte differentiation of 3T3-L1 cells, suggesting that CLA may be involved in the transcriptional regulation of adipogenic genes required for lipid accumulation in a PPAR $\gamma$-dependent and -independent manner (Miller et al., 2008).

TR4, a member of the nuclear receptor superfamily, regulates gene expression via binding to various TR4 response elements (TR4REs) in target gene promoters 
with highest affinity for DR1 element (Kim et al., 2003; Lee et al., 1995). TR4-deficient mice show reduced body fat mass with reduced TG accumulation in white adipose tissues (Kang et al., 2011; Kim et al., 2011).

Interestingly, various polyunsaturated fatty acids including $\gamma$-linoleic acid have been shown to stimulate TR4 transcriptional activity (Tsai et al., 2009). Moreover, Thiazolidinedione (TZD), a well-known PPAR $\gamma$ agonist, also promotes TR4 activity in the reporter gene assay (Xie et al., 2009). These previous reports strongly suggest that TR4 may function as a fatty acid sensor to modulate lipid homeostasis of our body (Xie et al., 2009). Thus, we hypothesized that CLA, a dietary component of milk products and ruminant meats, may participate in lipid homeostasis in adipocytes in part by modulation of TR4 activity. Here, we identified CLA is a functional modulator of TR4 and thus, suppresses TR4-induced perilipin promoter activity. Our findings suggest that TR4 is a key transcription factor which is negatively regulated by CLA during adipogenesis and this study will help to understand transcriptional network to control obesity-related diseases.

\section{Materials and Methods}

\section{Materials}

CLA and fatty acid free bovine serum albumin (BSA) was purchased from Sigma-aldrich. Rosiglitazone was purchased from Cayman. CLA was complexed to BSA to generate $32 \mathrm{mM}$ CLA stock solution with $1 \%$ fatty acid free BSA.

\section{Cell culture and adipocyte differentiation}

NIH-3T3, 3T3-L1 cells were maintained in Dulbecco's Modified Eagle's Medium (DMEM) with 10\% newborn calf serum and HEK293T cells were maintained in DMEM with $10 \%$ FBS. Adipocyte differentiation was described previously (Kim et al., 2010). Briefly, 2-day post confluent 3T3-L1 cells (designated day 0) were differentiated with the differentiation induction medium (MDI) containing $5 \mu \mathrm{g} / \mathrm{mL}$ insulin, $0.25 \mathrm{mM}$ 3-isobutyl-1-methylxanthine (IBMX) and $0.25 \mu \mathrm{M}$ dexamethasone in DMEM supplemented with $1 \%$ FBS.

\section{Plasmids and Luciferase assays}

Plasmids, pCMX-TR4, pCMX-4A4, and TR4RE-Luc (pGL-tk-(DR1) $)_{3}$-Luc) were described previously (Kim et al., 2003; Lee et al., 1995; Lee et al., 1997). The mouse perilipin promoter region consisting of -2000 to $+75 \mathrm{bp}$ was amplified by PCR from 3T3-L1 genomic DNA and cloned into pGL3-luciferase (Promega, USA) to generate pGL3-Plin-Luc. Three copies of synthesized DR1 element of the perilipin promoter and consensus DR1 (cDR1) were subcloned into pGL3- $\Delta 32 \mathrm{TK}$-Luc to create pGL3-Plin-DR1-Luc and pGL3-cDR1-Luc, respectively. Transfections were performed using SuperFect (Qiagen, USA) according to the manufacturer's instructions in HEK293T cells. After transfection, cells were incubated for $24 \mathrm{~h}$ in the absence of presence of CLA in DMEM supplement with $1 \%$ FBS and luciferase activities were measured in a Luciferase reporter assay system (Berthold). Relative luciferase activity (fold) was expressed based on the induction fold relative to transfection of an empty vector (set as 1-fold) and the results were expressed as means \pm SD of three separate experiments.

\section{Reverse transcriptase PCR (RT-PCR) and gel shift assay}

RNA isolation, cDNA synthesis and RT-PCR were performed as previously described (Kim et al., 2010). The sequences of TR4, aP2, and 36B4 primers used for RTPCR were described previously (Cariou et al., 2006; Kim et al., 2003). Primer sequences for perilipin gene were: sense; 5'-CTCTGGGAAGCATCGAGAA-3' and antisense; 5'-GATCCACATGGCCAGAGAGT-3'. Gel shift assay was performed as described previously (Kim et al., 2003). Briefly, TR4 was in vitro synthesized using a T7-quick coupled TNT reticulocyte lysate system (Promega). The following oligomers were used for gel shift assays: cDR1 (5'-GATCTCTCTAGGTCAAAGGTCAATTTC-3'), PlinDR1 (5'-GATCCTTGTCACCTTTCACCCACAT-3') and mutant Plin-DR1 (5'-GATCCCCTTGTCAGGACGATCCCACATCC-3').

\section{Results}

\section{CLA increased TR4 expression in 3T3-L1 adipo- cytes}

TR4-deficient mice have less fat mass with reduced TG accumulation. In agreement with this report, we recently found that TR4 regulates lipid accumulation in 3T3-L1 adipocytes via regulation of FATP1 expression. To investigate the possible involvement of TR4 in CLA effect on adipocyte biology, we added 20,40 or $80 \mu \mathrm{M}$ CLA together with standard adipogenic stimuli to 2-day post confluent 3T3-L1 cells (designated day 0) and CLA was continuously treated to the differentiating cells until day 6 of differentiation. Since CLA has been shown to inhibit 
adipogenesis, we determined effect of CLA on TR4 expression on day 6 using RT-PCR. Consistent with previous report, CLA significantly reduced expression of perilipin gene which is important for TG accumulation in adipocytes in a dose-dependent manner (Fig. 1). In contrast, TR4 expression was slightly increased by CLA treatment. In addition, mRNA levels of aP2, a well-known adipogenic marker gene, in CLA-treated cells were similar to that of 3T3-L1 cells treated with vehicle.

\section{CLA specifically modulates TR4 transcriptional activity}

PPAR $\gamma$ is a major transcription factor which plays a critical role in adipocyte differentiation and lipid metabolisms via regulation of various lipogenic genes. Previous reports have shown that CLA acts as a partial agonist of PPAR $\gamma$ in the absence of PPAR $\gamma$ ligand (Evans et al., 2010). To investigate whether CLA affects TR4 transcriptional activity, we compared effect of CLA on TR4 and PPAR $\gamma$ transactivation using reporter gene fused with 3 copies of cDR1 (pGL3-cDR1-Luc). As shown in Fig. 2A, transfection of PPAR $\gamma / \mathrm{RXR} \alpha$ into HEK293T cells increased the activity of the reporter gene in the presence of $200 \mu \mathrm{M}$ rosiglitazone. In agreement with previous report, CLA can also activate PPAR $\gamma$ activity. In contrast, CLA dramatically suppressed TR4 transcriptional activity in a dose-dependent manner. To further determine whether CLA acts as a negative TR4 regulator independently of DR1 sequences, we performed reporter gene assays using 2 different reporter genes fused with different DR1s (pGL3-Plin-DR1-Luc and pGL3-TR4RE -Luc). As shown in Fig 2B, CLA inhibited TR4-induced activities of both

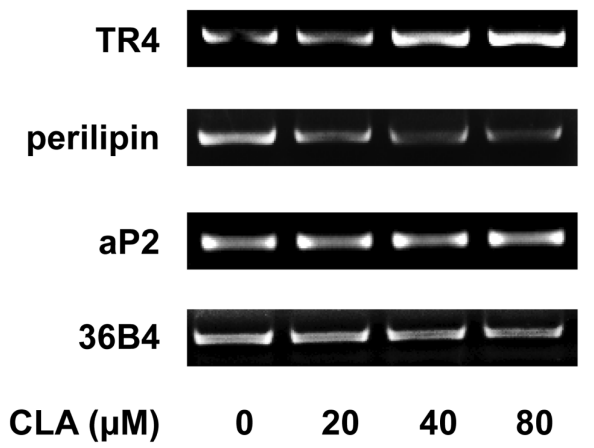

Fig. 1. Effect of CLA on adipogenic gene expression in 3T3Ll adipocytes. A. 3T3-L1 cells were treated with different doses of CLA $(0,20,40$, and $80 \mu \mathrm{M})$ from day 0 to day 6 after induction of differentiation. On day 6 , mRNA levels of aP2, TR4, and perilipin from vehicle- or CLAtreated adipocytes were determined by RT-PCR. Representative RT-PCR data shown here is from 3 independent experiments with similar results. reporter genes in a dose-dependent manner. This negative effect of CLA on TR4 transcriptional activity in a DR1 sequence-independent manner suggests that CLA may be a functional TR4 modulator.

\section{TR4 induces promoter activity via binding to a} DR1 located at the perilipin promoter

TR4 has been implicated in lipid metabolism and TR4deficient mice have reduced fat mass with smaller size of adipocytes (Kang et al., 2011; Kim et al., 2011). Furthermore, we also showed that TR4 facilitated lipid accumulation in 3T3-L1 adipocytes via regulation of FATP1. Since CLA has been known to inhibit adipogenesis in part by inhibition of perilipin gene (Chung et al., 2005) and TR4 activity is suppressed by CLA, it is possible that CLA effect on expression perilipin gene may be through inhibition of TR4 activity. Thus, we first determined whether TR4 could regulate the promoter activity of perilipin gene in HEK293T cells using a reporter gene fused with perilipin 5' promoter region (pGL3-Plin-Luc). As shown in Fig. 3A, TR4 induced perilipin promoter activity up to about 10-60 fold relative to transfection of empty vector. In contrast, 4A4, a TR4 mutant whose DNA binding domain was replaced by that of androgen receptor, did not induce luciferase activity of the pGL3-Plin-Luc, suggesting that TR4 may induce perilipin promoter activity via direct binding to the perilipin promoter. Since a previous study revealed the presence of a DR1 (GGGTGA A AGGTGA), a putative TR4RE, in perilipin promoter (Arimura et al., 2004) and TR4 transactivated pGL3-PlinDR1-Luc (Fig. 2B), we performed a gel shift assay to determine whether TR4 can bind to this DR1 sequence (Plin-DR1). As shown in Fig. 3B, in vitro-translated TR4 formed a complex with $\left[{ }^{32} \mathrm{P}\right]$-labeled DR1 sequence. Furthermore excess of cold Plin-DR1 but not of mutant PlinDR1 abolished TR4/Plin-DR1 complex in a dose-dependent manner. In addition, addition of anti-TR4 antibody resulted in supershift of TR4/Plin-DR1 complex. To test whether CLA suppression of perilipin expression via inhibition of TR4 transcriptional activity, we performed the luciferase assay using reporter gene fused with perilipin promoter. When CLA was added to the HEK293T cells transfected with TR4, it reduced TR4 transactivation of pGL3-Plin-Luc in a dose-dependent manner (Fig. 3C), suggesting that CLA may inhibit perilipin gene expression via downregulation of TR4 activity. TR4 is a member of the nuclear receptor superfamily and a unique nuclear receptor in that it has broad affinity for various DRs with different numbers of spacer nucleotide. Binding 

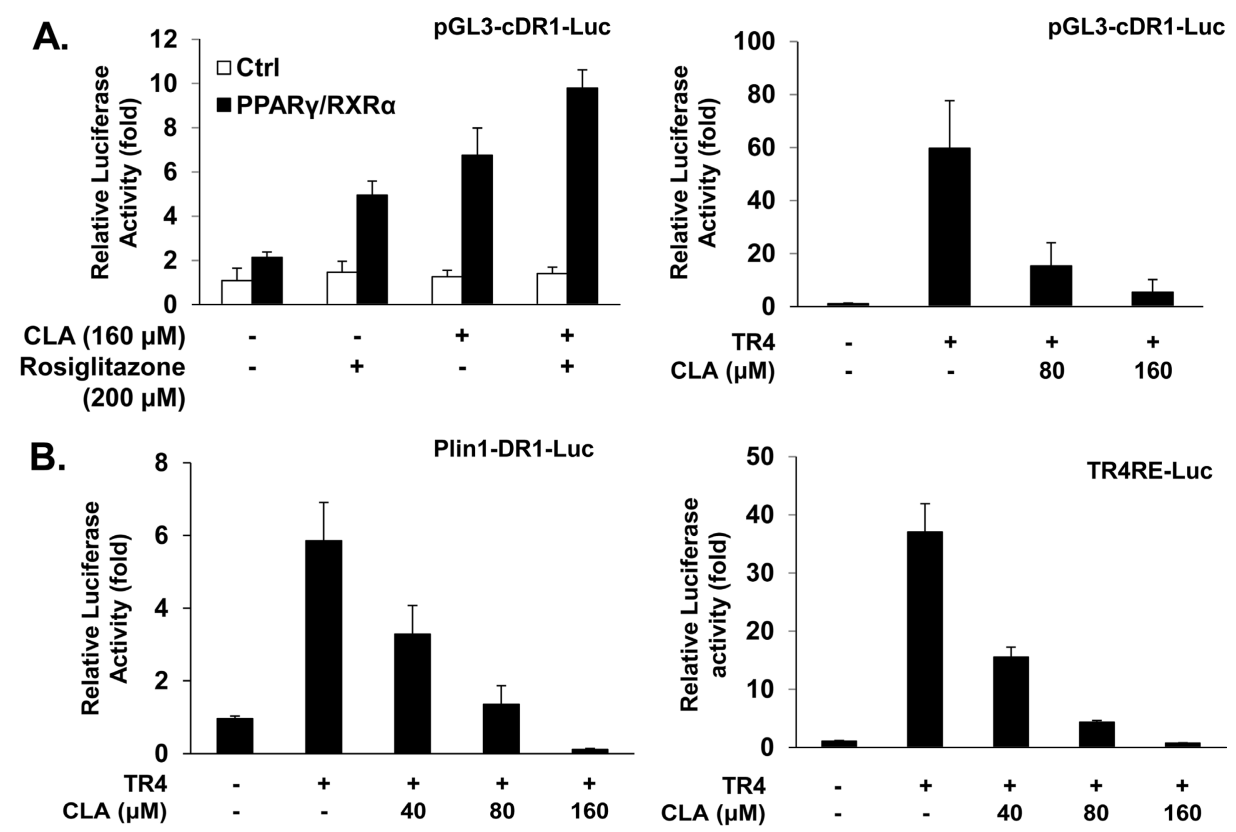

Fig. 2. CLA suppression of TR4 transcriptional activity. A. Reporter gene (pGL3-cDR1-Luc, $300 \mathrm{ng}$ ) was co-transfected with pSG5PPAR $\gamma$, pCMX-RXR $\alpha$ or pCMX-TR4 (100 ng each) into HEK293T cells. Cells were treated with rosiglitazone $(200 \mu \mathrm{M})$ or CLA $(80$ or $160 \mu \mathrm{M})$ in 1\% FBS for $24 \mathrm{~h}$ as indicated. B. HEK293T cells were transfected with pCMX-TR4 (100 ng) and indicated reporter genes $(300 \mathrm{ng}$ ) and then treated with different dosage of CLA in 1\% FBS for $24 \mathrm{~h}$ as indicated. The results were expressed as means $\pm \mathrm{SD}$ of three separate experiments.

affinity of the nuclear receptors for their cognate response elements could be influenced by agonist/antagonist. Since CLA modulates TR4 transcriptional activity, we investigated whether CLA could affect TR4 binding affinity for cDR1, which is known as the ideal TR4RE. In a gel shift assay, in vitro translated TR4 protein formed a specific complex with $\left[{ }^{32} \mathrm{P}\right]$-labeled cDR1 (Fig. 3D). When CLA was added together with in vitro translated TR4, the TR4 binding affinity for cDR1 was obviously reduced by CLA treatment, suggesting that CLA inhibition of TR4 transcriptional activity may be in part via reduction of TR4 binding affinity for TR4RE. Previous studies have shown that CLA is involved in cell signaling pathways (Chung et al., 2005). Various signals are frequently involved in signaling pathway of nuclear receptor family including TR4. TR4 phosphorylation by mitogen-activated protein kinase (MAPK) has shown to inhibit TR4 transcriptional activity (Huq et al., 2006). Since activation of MAPK in differentiating adipocytes has been reported to inhibit adipocyte differentiation with reduced lipid accumulation (Aouadi et al., 2006; Bost et al., 2005), we determined whether inhibitory effect of CLA on TR4 transactivation is via MAPK or other signalings using inhibitors of various signals. As observed in above results, CLA suppressed TR4 transactivation of pGL3-Plin-Luc in HEK293T cells (Fig. 3E). Inhibition of PKC or Akt using their cognate inhibitors (GF 109203X or LY294002, respectively) reduced suppressive effect of CLA on TR4 transactivation whereas inhibition of MAPK by PD98059 has no effect on CLA inhibition of TR4 activity.

\section{CLA inhibits TR4-induced perilipin expression in 3T3-L1 adipocytes}

Since TR4 induced perilipin promoter activity via direct binding to Plin-DR1, we then determined mRNA levels of perilipin gene in 3T3-L1 cells stably overexpressing TR4 to evaluate TR4 effect on expression of perilipin gene. RT-PCR analysis showed that perilipin gene expression was increased during adipocyte differentiation (Fig. 4A). Moreover, overexpression of TR4 in 3T3-L1 adipocytes resulted in further increase of perilipin expression as compared with the control adipocytes stably transfected with empty plasmid, indicating that TR4 activates perilipin expression. To examine the effect of CLA on TR4-induced perilipin expression, TR4 was transiently transfected in NIH-3T3 cell. Perilipin expression was significantly induced by TR4. However, when CLA (160 $\mu \mathrm{M})$ was added to the cell transfected with TR4, TR4induced perilipin gene expression was reduced by CLA treatment (Fig. 4B). To further determine the effect of CLA on TR4 induction of perilipin gene in adipocytes, we determined the effect of CLA on TR4-induced peril- 
A.

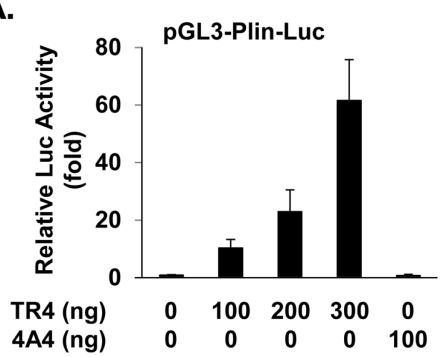

D.

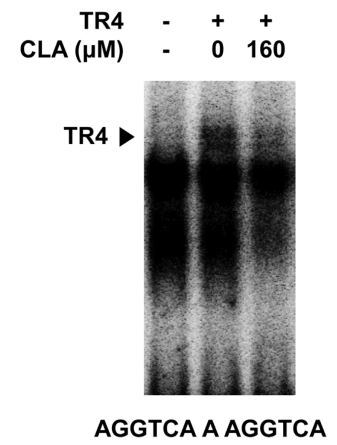

B.

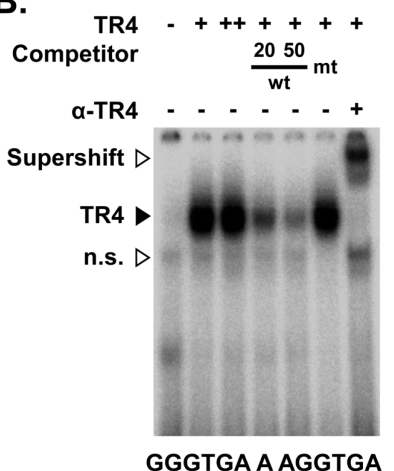

E.

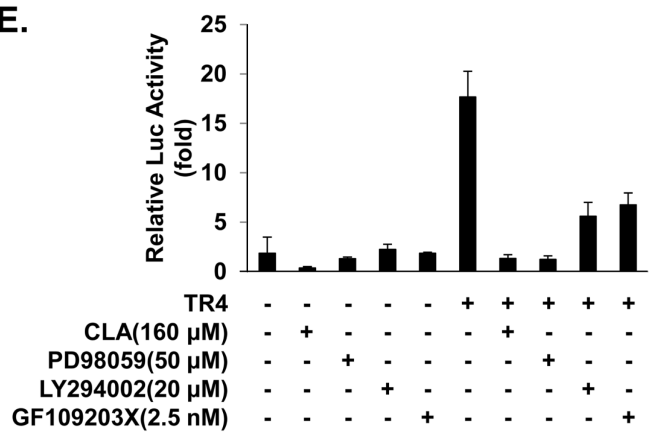

Fig. 3. TR4 regulates perilipin promoter activity by direct binding to TR4RE. A. Reporter gene fused with perilipin promoter (pGL3-Plin-Luc, $300 \mathrm{ng}$ ) were co-transfected with pCMX-TR4 (100, 200 and $300 \mathrm{ng}$ ) or pCMX-4A4 (100 ng) into HEK293T cells. Transfected cells were incubated in $1 \%$ FBS for $24 \mathrm{~h}$ and then harvested to measure luciferase activity. B. Gel shift assay was performed with in vitro translated TR4 using [ $\left.{ }^{32} \mathrm{P}\right]$-labeled Plin-DR1. Twenty and fifty molar excesses of unlabeled wild type (wt) or mutated (mt) oligonucleotides were added as competitors. Anti-TR4 antibody was added as indicated. The retarded and supershifted bands are indicated by closed and open arrowhead respectively. C. Reporter gene (pGL3-Plin-Luc, 300 ng) was cotransfected with pCMX-TR4 (100 ng) into HEK293T cells. Transfected cells were incubated with increasing amount of CLA in $1 \%$ FBS for $24 \mathrm{~h}$ and then harvested to measure luciferase activity. D. Gel shift assay was performed with in vitro translated TR4. The $\left[{ }^{32} \mathrm{P}\right]$-labeled cDR1 was used as a probe. CLA $(160 \mu \mathrm{M})$ was added as indicated. The retarded complex is indicated by closed arrowhead. E. HEK293T cells were transfected with pCMX-TR4 (100 ng) and pGL3-Plin-Luc (300 ng). Cells were pretreated with GF109203X $(2.5 \mathrm{nM})$, PD98059 $(25 \mu \mathrm{M})$ or LY294002 $(20 \mu \mathrm{M})$ for $1 \mathrm{~h}$ and then incubated with $160 \mu \mathrm{M}$ CLA for $24 \mathrm{~h}$ in $1 \%$ FBS as indicated.

ipin expression using 3T3-L1 preadipocytes stably expressing TR4. As expected, RT-PCR analysis showed that mRNA levels of perilipin gene was induced by TR4 in day 6 adipocytes as compared with control adipocytes (Fig. 4C). Consistent with above data, when $80 \mu \mathrm{M}$ CLA was added together with adipogenic stimuli, TR4 effect on perilipin gene expression was reduced.

\section{Discussion}

Inhibitory effect of CLA on lipid metabolism in adipocytes has been reported in cellular and animal models (Clement et al., 2002; Granlund et al., 2003). However, the regulatory cascades of CLA leading to this preventive effect on adipogenesis remain unclear. Recent reports have shown that TR4-deficient mice exhibit markedly reduced white fat masses with smaller sizes of adipocytes (Kang et al., 2011; Kim et al., 2011). In parallel, we also found that overexpression of TR4 in 3T3-L1 adipocytes promoted lipid accumulation. In this study, we found that TR4 induces perilipin gene expression through binding to TR4RE located within the perilipin promoter and TR4 transactivation of perilipin promoter is inhibited by CLA. Perilipin, a lipid-droplet associated protein, coats lipid droplets in differentiating adipocytes and restricts the access of cytosolic lipases to lipid droplets, resulting in promotion of triacylglycerol storage (Brasaemle et al., 2000). Early studies have shown that TR4 could modulate gene expression via binding to DR1 element although TR4 has broad affinity for variable DRs with different spacer sequences from 0 to 6 nucleotides (DR0-DR6) (Lee et al., 1995; Lee et al., 1997; Lee et al., 1999). Here, we show that TR4 regulates perilipin gene expression through DR1 element (Plin-TR4RE) located in the perilipin promoter. PPAR $\gamma$ is also able to bind the same DNA response element for induction of perilipin gene (Arimura 


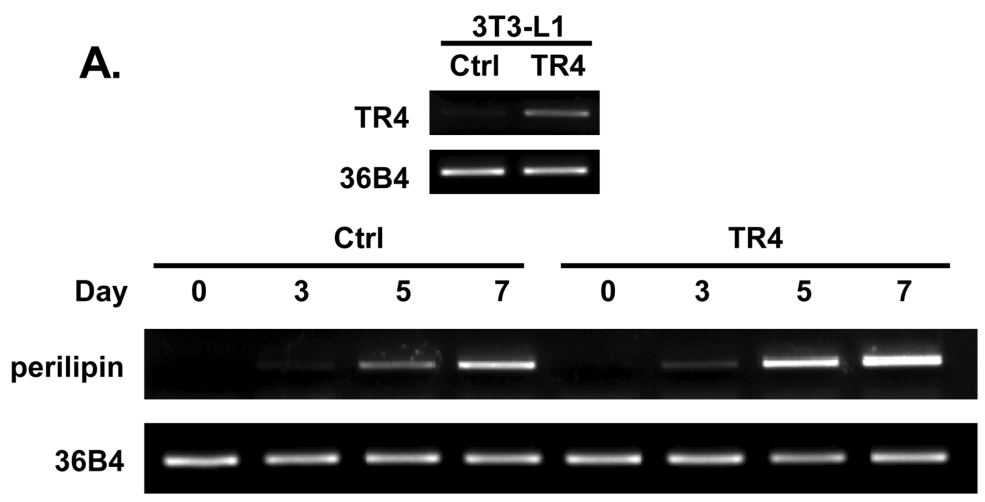

B.

TR4

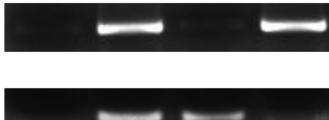

perilipin

aP2

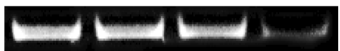

36B4

TR4

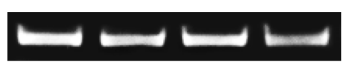

CLA (160 $\mu \mathrm{M})$

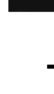

C.

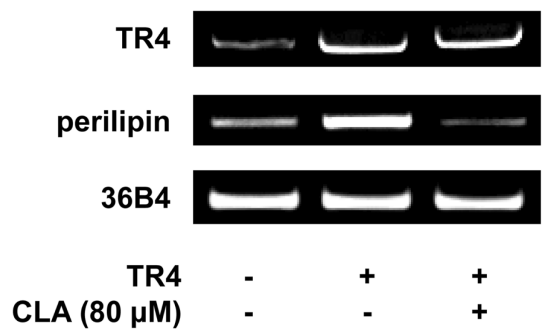

Fig. 4. TR4 regulates perilipin gene expression. A. Total RNA was extracted from 3T3-L1 cells stably overexpressing TR4 or control cells transfected with empty plasmid on the indicated days after induction. Perilipin expression was measured by RT-PCR. Representative RT-PCR data shown here is from 3 independent experiments with similar results. 36B4 was used as a loading control. B. NIH-3T3 cells were transiently transfected with pCMX-TR4 or empty plasmid. Cells were treated with $160 \mu \mathrm{M}$ CLA for $24 \mathrm{~h}$ in $1 \%$ FBS as indicated. mRNA levels of TR4, perilipin, aP2, and 36B4 from vehicle- or CLA-treated NIH-3T3 cells were determined by RT-PCR. C. Total RNA was extracted from 3T3-L1 cells stably overexpressing TR4 or control cells with vehicle or 80 $\mu \mathrm{M}$ CLA from day 0 to 6 . Perilipin expression was measured by RT-PCR. Representative RT-PCR data shown here is from 3 independent experiments with similar results. 36B4 was used as a loading control.

et al., 2004). Early studies suggested that CLA can suppress ligand-activated PPAR $\gamma$ but not the basal activity of PPAR $\gamma$ (Kennedy et al., 2008). Other studies showed that CLA plays a role as an activator of PPAR $\gamma$ and CLA increases reporter gene activity with PPAR $\gamma$ agonist (Evans et al., 2010; Miller et al., 2008). Previous studies have demonstrated that a variety of polyunsaturated fatty acids participate in TR4 transactivation (Tsai et al., 2009). Although further study is required to test the possibility of CLA as a TR4 inverse agonist, CLA plays a role as a functional modulator of TR4 to inhibit TR4 transcriptional activity. Since CLA effect on PPAR $\gamma$ activity was conflicting in several reports, it is plausible that CLA effect on TR4 activity could be variable when different TR4REs are used. However, we found that CLA consistently inhibited TR4 transcriptional activity when we used different reporter genes fused with different DR1 sequences, indicating that CLA is a functional modulator of TR4 activity. It has been reported that various signals such as MAPK/extracellular signal-related kinase (ERK) plays an important role as a signaling mediator for various nuclear receptors including TR4 (Chen et al., 2005; Huq et al., 2006). Early study showed that TR4 can be phosphorylated by MAPK and this phosphorylation reduces TR4 activity (Huq et al., 2006). Interestingly, CLA is involved in various signaling pathways including MAPK/ ERK (Chung et al., 2005). CLA regulation of cell signals such as MAPK/ERK and PI3K/Akt may cause inhibition of TR4 activity. In line with this notion, CLA inhibition of TR4 transcriptional activity was reduced when inhibitors of PI3K/Akt and PKC but not of MAPK were added together with CLA.

Together, we demonstrated that TR4 induces perilipin gene expression through activation of perilipin promoter activity by direct binding to TR4RE and this TR4 stimulation of perilipin promoter activity could be abolished by CLA treatment. Thus, pharmacological manipulation of TR4 activities may be a promising therapeutic approach for treatment of obesity-related diseases. 


\section{Acknowledgement}

This work was supported by National Research Foundation of Korea Grant funded by the Korean Government (KRF-2006-312-C00392).

\section{References}

1. Alibin, C. P., Kopilas, M. A., and Anderson, H. D. (2008) Suppression of cardiac myocyte hypertrophy by conjugated linoleic acid: role of peroxisome proliferator-activated receptors alpha and gamma. J. Biol. Chem. 283, 10707-10715.

2. Aouadi, M., Laurent, K., Prot, M., Le Marchand-Brustel, Y., Binetruy, B., and Bost, F. (2006) Inhibition of p38MAPK increases adipogenesis from embryonic to adult stages. Diabetes 55, 281-289.

3. Arimura, N., Horiba, T., Imagawa, M., Shimizu, M., and Sato, R. (2004) The peroxisome proliferator-activated receptor gamma regulates expression of the perilipin gene in adipocytes. J. Biol. Chem. 279, 10070-10076.

4. Bost, F., Aouadi, M., Caron, L., and Binetruy, B. (2005) The role of MAPKs in adipocyte differentiation and obesity. Biochimie 87, 51-56.

5. Brasaemle, D. L., Rubin, B., Harten, I. A., Gruia-Gray, J., Kimmel, A. R., and Londos, C. (2000) Perilipin A increases triacylglycerol storage by decreasing the rate of triacylglycerol hydrolysis. J. Biol. Chem. 275, 38486-38493.

6. Cariou, B., van Harmelen, K., Duran-Sandoval, D., van Dijk, T. H., Grefhorst, A., Abdelkarim, M., Caron, S., Torpier, G., Fruchart, J. C., Gonzalez, F. J., Kuipers, F., and Staels, B. (2006) The farnesoid $X$ receptor modulates adiposity and peripheral insulin sensitivity in mice. J. Biol. Chem. 281, 11039-11049.

7. Chen, S., Operana, T., Bonzo, J., Nguyen, N., and Tukey, R. H. (2005) ERK kinase inhibition stabilizes the aryl hydrocarbon receptor: implications for transcriptional activation and protein degradation. J. Biol. Chem. 280, 4350-4359.

8. Chung, S., Brown, J. M., Sandberg, M. B., and McIntosh, M. (2005) Trans-10,cis-12 CLA increases adipocyte lipolysis and alters lipid droplet-associated proteins: role of mTOR and ERK signaling. J. Lipid Res. 46, 885-895.

9. Clement, L., Poirier, H., Niot, I., Bocher, V., Guerre-Millo, M., Krief, S., Staels, B., and Besnard, P. (2002) Dietary trans-10,cis-12 conjugated linoleic acid induces hyperinsulinemia and fatty liver in the mouse. J. Lipid Res. 43, 14001409.

10. Evans, N. P., Misyak, S. A., Schmelz, E. M., Guri, A. J., Hontecillas, R., and Bassaganya-Riera, J. (2010) Conjugated linoleic acid ameliorates inflammation-induced colorectal cancer in mice through activation of PPARgamma. J. Nutr. 140, 515-521.

11. Granlund, L., Juvet, L. K., Pedersen, J. I., and Nebb, H. I. (2003) Trans10, cis12-conjugated linoleic acid prevents triacylglycerol accumulation in adipocytes by acting as a PPARgamma modulator. J. Lipid Res. 44, 1441-1452.
12. Gregor, M. F. and Hotamisligil, G. S. (2007) Thematic review series: Adipocyte Biology. Adipocyte stress: the endoplasmic reticulum and metabolic disease. J. Lipid Res. 48, 19051914.

13. Guilherme, A., Virbasius, J. V., Puri, V., and Czech, M. P. (2008) Adipocyte dysfunctions linking obesity to insulin resistance and type 2 diabetes. Nat. Rev. Mol. Cell Biol. 9, 367-377.

14. Ha, Y. L., Storkson, J., and Pariza, M. W. (1990) Inhibition of benzo(a)pyrene-induced mouse forestomach neoplasia by conjugated dienoic derivatives of linoleic acid. Cancer Res. 50, 1097-1101.

15. Huq, M. D., Gupta, P., Tsai, N. P., and Wei, L. N. (2006) Modulation of testicular receptor 4 activity by mitogen-activated protein kinase-mediated phosphorylation. Mol. Cell Proteomics 5, 2072-2082.

16. Kang, H. S., Okamoto, K., Kim, Y. S., Takeda, Y., Bortner, C. D., Dang, H., Wada, T., Xie, W., Yang, X. P., Liao, G., and Jetten, A. M. (2011) Nuclear orphan receptor TAK1/TR4deficient mice are protected against obesity-linked inflammation, hepatic steatosis, and insulin resistance. Diabetes $\mathbf{6 0}$, 177-188.

17. Kang, K., Liu, W., Albright, K. J., Park, Y., and Pariza, M. W. (2003) trans-10,cis-12 CLA inhibits differentiation of 3T3L1 adipocytes and decreases PPAR gamma expression. Biochem. Biophys. Res. Commun. 303, 795-799.

18. Kennedy, A., Chung, S., LaPoint, K., Fabiyi, O., and McIntosh, M. K. (2008) Trans-10, cis-12 conjugated linoleic acid antagonizes ligand-dependent PPARgamma activity in primary cultures of human adipocytes. J. Nutr. 138, 455-461.

19. Kennedy, A., Overman, A., Lapoint, K., Hopkins, R., West, T., Chuang, C. C., Martinez, K., Bell, D., and McIntosh, M. (2009) Conjugated linoleic acid-mediated inflammation and insulin resistance in human adipocytes are attenuated by resveratrol. J. Lipid Res. 50, 225-232.

20. Kershaw, E. E. and Flier, J .S. (2004) Adipose tissue as an endocrine organ. J. Clin. Endocrinol. Metab. 89, 2548-2556

21. Kim, E., Liu, N. C., Yu, I. C., Lin, H. Y., Lee, Y. F., Sparks, J. D., Chen, L. M., and Chang, C. (2011) Metformin Inhibits Nuclear Receptor TR4-Mediated Hepatic Stearoyl-Coenzyme A Desaturase 1 Gene Expression With Altered Insulin Sensitivity. Diabetes DOI: 10.2337/db2310-0393.

22. Kim, E., Xie, S., Yeh, S. D., Lee, Y. F., Collins, L. L., Hu, Y. C., Shyr, C. R., Mu, X. M., Liu, N. C., Chen, Y. T., Wang, P. H., and Chang, C. (2003) Disruption of TR4 orphan nuclear receptor reduces the expression of liver apolipoprotein E/CI/C-II gene cluster. J. Biol. Chem. 278, 46919-46926.

23. Kim, S. J., Choi, H., Jung, C. H., Park, S. S., Cho, S. R., Oh, S., and Kim, E. (2010) Calcium Mobilization Inhibits Lipid Accumulation During the Late Adipogenesis via Suppression of PPAR gamma and LXR alpha Signalings. Korean J. Food Sci. Ani. Resour. 30, 787-794.

24. Larsen, T. M., Toubro, S., and Astrup, A. (2003) Efficacy and safety of dietary supplements containing CLA for the treatment of obesity: evidence from animal and human studies. J. Lipid Res. 44, 2234-2241. 
25. Lee, H. J., Lee, Y., Burbach, J. P., and Chang, C. (1995) Suppression of gene expression on the simian virus 40 major late promoter by human TR4 orphan receptor. A member of the steroid receptor superfamily. J. Biol. Chem. 270, 3012930133.

26. Lee, K. N., Kritchevsky, D., and Pariza, M. W. (1994) Conjugated linoleic acid and atherosclerosis in rabbits. Atherosclerosis 108, 19-25.

27. Lee, Y. F., Pan, H. J., Burbach, J. P., Morkin, E., and Chang, C. (1997) Identification of direct repeat 4 as a positive regulatory element for the human TR4 orphan receptor. A modulator for the thyroid hormone target genes. J. Biol. Chem. 272, 12215-12220.

28. Lee, Y. F., Young, W. J., Lin, W. J., Shyr, C. R., and Chang, C. (1999) Differential regulation of direct repeat 3 vitamin D3 and direct repeat 4 thyroid hormone signaling pathways by the human TR4 orphan receptor. J. Biol. Chem. 274, 16198-16205.

29. Miller, J. R., Siripurkpong, P., Hawes, J., Majdalawieh, A., Ro, H. S., and McLeod, R. S. (2008) The trans-10, cis-12 isomer of conjugated linoleic acid decreases adiponectin assembly by PPARgamma-dependent and PPARgamma-independent mechanisms. J. Lipid Res. 49, 550-562.
30. Rosen, E. D. and Spiegelman, B. M. (2006) Adipocytes as regulators of energy balance and glucose homeostasis. Nature 444, 847-853

31. Sakuma, S., Nishioka, Y., Imanishi, R., Nishikawa, K., Sakamoto, H., Fujisawa, J., Wada, K., Kamisaki, Y., and Fujimoto, Y. (2010) cis9, trans11-Conjugated linoleic acid differentiates mouse 3T3-L1 preadipocytes into mature small adipocytes through induction of peroxisome proliferator-activated receptor gamma. J. Clin. Biochem. Nutr. 47, 167-173.

32. Shi, Y. and Burn, P. (2004) Lipid metabolic enzymes: emerging drug targets for the treatment of obesity. Nat. Rev. Drug Discov. 3, 695-710.

33. Tsai, N.P., Huq, M., Gupta, P., Yamamoto, K., Kagechika, H., and Wei, L. N. (2009) Activation of testicular orphan receptor 4 by fatty acids. Biochim. Biophys. Acta 1789, 734740.

34. Xie, S., Lee, Y. F., Kim, E., Chen, L. M., Ni, J., Fang, L. Y., Liu, S., Lin, S. J., Abe, J., Berk, B., Ho, F. M., and Chang, C. (2009) TR4 nuclear receptor functions as a fatty acid sensor to modulate CD36 expression and foam cell formation. $P$. Natl. Acad. Sci. USA 106, 13353-13358.

(Received 2011.4.26/Revised 2011.5.24/Accepted 2011.5.25) 